Article

\title{
Field Fluctuations and Casimir Energy of 1D-Fermions
}

\author{
Manuel Donaire $\mathbb{D}^{\mathbb{D}}$, José María Muñoz-Castañeda ${ }^{\mathbb{D}}$, Luis Miguel Nieto * ${ }^{\mathbb{D}}$ and \\ Marcos Tello-Fraile \\ Departamento de Física Teórica, Atómica y Óptica and IMUVA, Universidad de Valladolid, \\ 47011 Valladolid, Spain; manuel.donaire@uva.es (M.D.); jose.munoz.castaneda@uva.es (J.M.M.-C.); \\ marcos.tello@uva.es (M.T.-F.) \\ * Correspondence: luismiguel.nieto.calzada@uva.es
}

Received: 15 April 2019; Accepted: 4 May 2019; Published: 7 May 2019

\begin{abstract}
We investigate the self-adjoint extensions of the Dirac operator of a massive one-dimensional field of mass $m$ confined in a finite filament of length $L$. We compute the spectrum of vacuum fluctuations of the Dirac field under the most general dispersionless boundary conditions. We identify its edge states in the mass gap within a set of values of the boundary parameters, and compute the Casimir energy of the discrete normal modes. Two limit cases are considered, namely, that of light fermions with $m L \ll 1$, and that of heavy fermions for which $m L \gg 1$. It is found that both positive and negative energies are obtained for different sets of values of the boundary parameters. As a consequence of our calculation we demonstrate that the sign of the quantum vacuum energy is not fixed for exchange-symmetric plates (parity-invariant configurations), unlike for electromagnetic and scalar fields.
\end{abstract}

Keywords: vacuum fluctuations; boundary conditions; Dirac field; Casimir effect

\section{Introduction}

Over the last two decades, the application of the theory of self-adjoint extensions of elliptic operators to problems of quantum physics has motivated an intensive research activity. In particular, in the seminal paper of Asorey, Marmo and Ibort [1], the authors have reformulated the classical theory of self-adjoint extensions pioneered by Von Neumann. They have studied the properties of the Laplace-Beltrami operator over a compact manifold with boundary, in terms of physically meaningful boundary data. In turn, Ref. [1] was based on the previous general result obtained by G. Grubb [2]. Later on, the results in Ref. [1] were used in Refs. [3-7] to develop the theory of scalar quantum fields confined in domains with boundaries. In particular, those works have applied the theory of self-adjoint extensions to the computation of the so-called Casimir energy of scalar fields [8,9]. On the other hand, Ibort et al. have developed the theory of local self-adjoint extensions for Dirac-type operators, and have studied applications in condensed matter [10-12]. More recent works have shown how the theory of self-adjoint extensions can be used to describe physical systems confined to cavities [13,14]. In addition, the authors of Ref. [15] have applied the theory of self-adjoint extensions for Dirac-type operators to describe fundamental phenomena in topological insulators, such as edge states. The quantum vacuum energy for Dirac fields has been largely studied previously, for the case that the self-adjoint extension of the Dirac operator that represents the interaction of the quantum field with the boundary is given by the M.I.T. bag boundary condition in its most general form (see Refs. [16-19]).

In this paper, we compute the spectrum of vacuum fluctuations of a massive Dirac field confined in a one-dimensional filament, and study its Casimir energy. The remainder of the article is organised as follows. In Section 2 we describe the operators of our fermionic system, their basic properties, and the spinor representation. In Section 3 we deal with the spectrum of fluctuations for the Dirac 
field, starting with the construction of the boundary spinors and finalizing with the computation of normal modes and edge states. In Section 4 we compute the Casimir energy of a massive Dirac field in two limit cases, namely for light and heavy fermions. In addition, at the end of Section 4 we study the sign of the Casimir energy for parity-invariant cases. We summarise our conclusions in Section 5.

\section{Fundamentals of the Approach}

The Lagrangian density of the free massive Dirac field, $\Psi(x, t)$, in one spatial dimension is $\mathcal{L}=\bar{\Psi}\left(i \gamma^{\mu} \partial_{\mu}-m\right) \Psi$, with $\bar{\Psi}=\Psi^{\dagger} \gamma^{0}$, where $\Psi^{\dagger}$ is the transposed and conjugated of $\Psi$, from which the field equation reads

$$
\left(i \gamma^{\mu} \partial_{\mu}-m\right) \Psi=0,
$$

where the index $\mu$ takes the values $0,1, m$ is the mass and $\gamma^{\mu}$ are the Dirac matrices that form the Clifford algebra in 1D. If we consider the metric tensor of the form $g^{\mu v}=\operatorname{diag}(1,-1)$, then

$$
\left(\gamma^{0}\right)^{2}=-\left(\gamma^{1}\right)^{2}=\mathbb{I}, \quad\left\{\gamma^{0}, \gamma^{1}\right\}=0 .
$$

The Dirac Hamiltonian operator is

$$
H=-i \alpha \partial_{1}+m \gamma^{0}, \quad \text { with } \alpha=\gamma^{0} \gamma^{1},
$$

and Schrödinger's equation reads $i \partial_{t} \Psi=H \Psi$. Expanding $\Psi(x, t)$ in normal modes,

$$
\Psi(x, t)=\frac{1}{2 \pi} \int_{-\infty}^{\infty} e^{-i E t} \psi(x ; k) d k,
$$

where $\psi(x ; k)=\exp (i k x) u(k)$, the eigenvalue equation of the Dirac Hamiltonian for $\psi(x ; k)$ reads,

$$
H \psi=E \psi,
$$

which gives rise to the ordinary $2 \times 2$ eigenvalue equation for spinors,

$$
h(k) u(k)=E u(k), \quad h(k) \equiv k \alpha+m \gamma^{0} .
$$

Solutions to this equation have eigenvalues

$$
E_{ \pm}= \pm \omega, \quad \omega \equiv \sqrt{k^{2}+m^{2}}>0,
$$

and corresponding spinor eigenstates $u_{+}(k)$ for $E_{+}=\omega$ (particles), and $u_{-}(k)$ for $E_{-}=-\omega$ (anti-particles). It is of note that the exchange $k \rightarrow-k$ is obtained in momentum space with the following operations

$$
h(-k)=\gamma^{0} h(k) \gamma^{0}, \quad u_{ \pm}(-k)=\gamma^{0} u_{ \pm}(k) .
$$

With these standard results, considering $k>0$ in all the cases, we can distinguish four types of free particle or anti-particle eigenstates $\psi(x ; k)$, namely,

- $\quad$ Particles moving from left to right with $E=\omega$ and momentum $k, \psi_{+}(x ; k)=e^{i k x} u_{+}(k)$.

- Particles moving from right to left with $E=\omega$ and momentum $k, \psi_{+}(x ;-k)=e^{-i k x} \gamma^{0} u_{+}(k)$.

- Anti-particles moving from left to right with $E=\omega$ and momentum $k, \psi_{-}(x ;-k)=e^{-i k x} \gamma^{0} u_{-}(k)$.

- Anti-particles moving from right to left with $E=\omega$ and momentum $k, \psi_{-}(x ; k)=e^{i k x} u_{-}(k)$.

Note that the explicit expressions for the spinors $u_{ \pm}(k)$ depend on the choice of $\gamma$-matrices. 


\section{Spectrum of Fluctuations of the Dirac Field Confined in a Finite Filament}

\subsection{Construction of the Boundary Spinors and the Boundary Condition}

Let us consider the Dirac field confined to a one-dimensional filament of length $L$, for which $x \in \Omega=[-L / 2, L / 2]$, and its boundary $\partial \Omega$ consists of the points $x=L / 2$ and $x=-L / 2$. In addition, we will consider its dual geometry, where the confinement takes place in the semi-infinite intervals $x \in \widetilde{\Omega}=\{(-\infty,-L / 2] \cup[L / 2, \infty)\}$. Hereafter, we will refer to the former as Casimir geometry for its analogy with the geometry of the original Casimir setup, in which the electromagnetic field is confined between two perfectly conducting plates separated at distance $L$; and as dual Casimir geometry to the latter.

In this situation the Dirac Hamiltonian (3) is not essentially self-adjoint on the spaces $\Omega, \tilde{\Omega}$. The obstruction to the self-adjointness of the Dirac Hamiltonian is due to the non-trivial structure of the space of boundary spinors, and is given by the integration by parts formula (see Ref. [20])

$$
\langle\psi, H \phi\rangle-\langle H \psi, \phi\rangle=i \int_{\partial \Omega} \psi^{\dagger}(\mathbf{n} \cdot \boldsymbol{\alpha}) \phi d s, \quad \forall \psi, \phi \in \mathcal{L}^{2}(\Omega),
$$

where $\mathbf{n}$ is the outgoing normal unitary vector to $\partial \Omega, \alpha=\hat{\mathbf{x}} \alpha, \hat{\mathbf{x}}$ is a unitary vector along the $x$-axis, and $\psi, \phi$ are two independent spinors of a common mode $k$. Hereafter, for the sake of brevity, we will drop the dependence of $\psi$ and $\phi$ on the wave number $k$. It is of note that the boundary term is the current flow throughout the boundary. Thus self-adjointness of Dirac fields is equivalent to the charge conservation. Hence the role played by unitarity in the quantum scalar field over the finite interval is translated into charge conservation for the Dirac field confined over the finite line. For the Casimir geometry, the normal outgoing vector to the boundary is given by $\mathbf{n}=-\hat{\mathbf{x}}$ at $x=-L / 2$ and $\mathbf{n}=\hat{\mathbf{x}}$ at $x=+L / 2$, so the general boundary term reads

$$
\langle\psi, H \phi\rangle-\langle H \psi, \phi\rangle=i\left(\psi^{\dagger}(L / 2) \alpha \phi(L / 2)+\psi^{\dagger}(-L / 2) \alpha \phi(-L / 2)\right), \quad \forall \psi, \phi \in \mathcal{L}^{2}(\Omega) .
$$

Note that the boundary term for the dual Casimir geometry has the same expression with a global minus in the right hand side of (10) due to the switch in signs for $\mathbf{n}$. Hence what comes next extends to the dual Casimir geometry with no change. In addition, let us emphasise here that the arguments to build up the space of boundary data from the bundle of boundary spinors can be extended to any dimension of the physical space, i.e., $\mathbb{R}^{d} \times \Omega$.

In the sequel, we will follow chapter one of Ref. [20] for the construction of the boundary spinors. In the first place, since the $\mathbf{n} \alpha$ matrix satisfies $(\mathbf{n} \boldsymbol{\alpha})^{\dagger}=\mathbf{n} \alpha$, and $(\mathbf{n} \boldsymbol{\alpha})^{2}=\mathbb{I}$, the space $\mathcal{H}$ of boundary spinors (spinors on $\mathcal{L}^{2}(\Omega)$ restricted to $\partial \Omega$ ) can be written as the direct sum

$$
\mathcal{H}=\mathcal{H}_{+} \oplus \mathcal{H}_{-},
$$

where $\mathcal{H}_{ \pm}=\{\xi \in \mathcal{H} \mid(\mathbf{n} \cdot \boldsymbol{\alpha}) \xi= \pm \xi\}$. Hermiticity of $(\mathbf{n} \boldsymbol{\alpha})$ ensures that elements in $\mathcal{H}_{+}$are orthogonal to elements in $\mathcal{H}_{-}$. In addition, this ensures that any boundary spinor $\xi$ can be written in a unique way as

$$
\xi=\xi^{+}+\xi^{-}, \quad \xi^{+} \in \mathcal{H}_{+}, \quad \xi^{-} \in \mathcal{H}_{-} .
$$

This direct sum structure enables to write the boundary term in (9) in terms of the direct sum decomposition of $\left.\psi\right|_{\partial \Omega}=\xi_{1}^{+}+\xi_{1}^{-}$and $\left.\phi\right|_{\partial \Omega}=\xi_{2}^{+}+\xi_{2}^{-}$as

$$
\langle\psi, H \phi\rangle-\langle H \psi, \phi\rangle=i \int_{\partial \Omega}\left(\left(\xi_{1}^{+}\right)^{\dagger} \xi_{2}^{+}-\left(\xi_{1}^{-}\right)^{+} \xi_{2}^{-}\right) d s .
$$

The central theorem in [20] states that all the self-adjoint extensions that do not present non-local behaviour (Global boundary conditions that involve changes in the topology are not included in the central theorem in chapter one of Ref. [20]. Nevertheless, they are also meaningful from a physical 
point of view as it is explained in Refs. $[1,6,21])$ at the boundary are in one-to-one correspondence with the local unitary operators

$$
\widetilde{U}: \mathcal{H}_{+} \longrightarrow \mathcal{H}_{-},
$$

and the domain of the self-adjoint extension associated, $H^{\widetilde{U}}$ is given by

$$
\mathcal{D}\left(H^{\widetilde{U}}\right)=\left\{\psi \in \mathcal{L}^{2}(\Omega)|\psi|_{\partial \Omega}=\xi^{+}+\widetilde{U}\left(\xi^{+}\right)\right\} .
$$

This means that the self-adjoint extension $H^{\widetilde{U}}$ is determined by the domain of $\mathcal{L}^{2}$ spinors that satisfy the boundary condition

$$
\xi^{-}=\widetilde{U} \xi^{+}
$$

Taking now into account that $\left\{\gamma^{0}, \mathbf{n} \cdot \boldsymbol{\alpha}\right\}=0,\left(\gamma^{0}\right)^{\dagger}=\gamma^{0}$, and $\left(\gamma^{0}\right)^{2}=\mathbb{I}$,

$$
\gamma^{0}: \mathcal{H}_{+} \longrightarrow \mathcal{H}_{-}
$$

is an isomorphism that enables to write any unitary operator $\widetilde{U}: \mathcal{H}_{+} \longrightarrow \mathcal{H}_{-}$, as

$$
\widetilde{U}=U \gamma^{0},
$$

with $U$ being any unitary operator of $\mathcal{H}_{-}$such that $[U, \mathbf{n} \cdot \boldsymbol{\alpha}]=0$, or equivalently $\left\{U \gamma^{0}, \mathbf{n} \cdot \boldsymbol{\alpha}\right\}=0$.

Let us now particularise the previous results to the $(1+1)$ dimensional case with $\Omega=[-L / 2, L / 2]$. In such a case

$$
\mathbf{n} \cdot \boldsymbol{\alpha}= \begin{cases}\alpha, & x=L / 2, \\ -\alpha, & x=-L / 2,\end{cases}
$$

We can choose a basis in $\mathbb{R}^{2}$ such that $\alpha$ is diagonal, i.e.,

$$
\alpha=\sigma_{3}
$$

without loss of generality. This means that any spinor mode of wave number $k, \psi \in \mathcal{L}^{2}(\Omega)$, can be written as

$$
\psi=\left(\begin{array}{l}
\psi_{1}(x) \\
\psi_{2}(x)
\end{array}\right)=\psi_{1}(x) \mathbf{e}_{+}+\psi_{2}(x) \mathbf{e}_{-}, \quad \alpha \mathbf{e}_{ \pm}= \pm \mathbf{e}_{ \pm},
$$

where we omit again the dependence on $k$ for brevity, and $\left\{\mathbf{e}^{+}, \mathbf{e}^{-}\right\}$form an orthonormal basis for one-dimensional spinors. Looking at Equation (19), it is obvious that for the decomposition $\left.\psi\right|_{\partial \Omega}=\xi^{+}+\xi^{-}$we need to take into account that:

- At $x=-L / 2$ the components $\xi^{ \pm}$are determined by the eigenvalue equation $(-\alpha) \mathbf{v}= \pm \mathbf{v}$. This means that the $\mathbf{e}_{ \pm}$component of $\psi(-L / 2)$ contributes to $\xi^{\mp}$.

- At $x=L / 2$ the components $\xi^{ \pm}$are determined by the eigenvalue equation $\alpha \mathbf{v}= \pm \mathbf{v}$. This means that the $\mathbf{e}_{ \pm}$component of $\psi(L / 2)$ contributes to $\xi^{ \pm}$.

Hence, the boundary data in this case are

$$
\xi^{ \pm}=\left(\begin{array}{l}
\psi_{1}( \pm L / 2) \\
\psi_{2}(\mp L / 2)
\end{array}\right)
$$

and the set of local boundary conditions that give rise to self-adjoint extensions of the Dirac operator (3) in the finite interval $\Omega=[-L / 2, L / 2]$ read 


$$
\xi^{-}=\mathbb{U} \gamma_{0} \xi^{+},
$$

with $\mathbb{U} \in U(2)$ such that $[\mathbb{U}, \alpha]=0$ or, equivalently, $\left\{\mathbb{U} \gamma^{0}, \alpha\right\}=0$. Taking into account that any $\mathbb{U} \in U(2)$ can be written in terms of the Pauli matrices $\left\{\sigma_{1}, \sigma_{2}, \sigma_{3}\right\}$ as

$$
\mathbb{U}=e^{i \theta}\left[\cos \eta \mathbb{I}_{2 \times 2}+i \sin \eta n^{j} \sigma_{j}\right], \quad j=1,2,3,
$$

with $n_{j} \in \mathbb{R}, n_{1}^{2}+n_{2}^{2}+n_{3}^{2}=1, \theta \in[0,2 \pi], \eta \in[-\pi / 2, \pi / 2]$, and that $\alpha$ is given by (20), the only allowed matrices of $U(2)$ in (23) by the requirement $\left\{\mathbb{U} \gamma^{0}, \alpha\right\}=0$ are

$$
\mathbb{U}=e^{i \theta}\left(\cos \eta \mathbb{I}_{2 x 2} \pm i \sin \eta \sigma_{3}\right)=\left(\begin{array}{cc}
e^{i(\theta \pm \eta)} & 0 \\
0 & e^{i(\theta \mp \eta)}
\end{array}\right) .
$$

Taking into account that $\left\{\gamma^{0}, \alpha\right\}=0$ and the general properties of $\gamma^{0}$,

$$
\alpha=\sigma_{3} \Rightarrow \gamma^{0}=(-1)^{r} \sigma_{1}, \quad r=0,1,
$$

we find out

$$
\mathbb{U} \gamma^{0}=(-1)^{r}\left(\begin{array}{cc}
0 & e^{i(\theta \pm \eta)} \\
e^{i(\theta \mp \eta)} & 0
\end{array}\right)
$$

from which it holds that

$$
\psi_{1}(-L / 2)=(-1)^{r} e^{i(\theta \pm \eta)} \psi_{2}(-L / 2), \quad \psi_{1}(L / 2)=(-1)^{r} e^{i(\theta \mp \eta)} \psi_{2}(L / 2) .
$$

\subsection{Normal Modes}

In order to find out the non-trivial solutions of normal modes, we plug into Equation (23) a generic linear combination of plane-wave spinors. We choose $\alpha$ as in (20), and $\gamma^{0}=\sigma_{1}$ in (26). With this election, the free spinors $u_{ \pm}(k)$ defined in Equations (6) and (7) are explicitly given by

$$
u_{+}(k)=\left(\begin{array}{c}
\sqrt{\omega+k} \\
\sqrt{\omega-k}
\end{array}\right), \quad u_{-}(k)=\left(\begin{array}{c}
\sqrt{\omega-k} \\
-\sqrt{\omega+k}
\end{array}\right) .
$$

The general static solution with negative energy has the form (the spectrum of positive energy is in all equivalent)

$$
\psi_{-}(x)=V_{+} e^{i k x} u_{-}(k)+V_{-} e^{-i k x} \gamma^{0} u_{-}(k),
$$

where $V_{ \pm}$are constants to be determined. From the last equation we can immediately read out the components $\psi_{1}$ and $\psi_{2}$ that enter the boundary condition (23):

$$
\psi_{1}=V_{+} e^{i k x} \sqrt{\omega-k}-V_{-} e^{-i k x} \sqrt{\omega+k}, \quad \psi_{2}=-V_{+} e^{i k x} \sqrt{\omega+k}+V_{-} e^{-i k x} \sqrt{\omega-k}
$$

From Equation (31) it is straightforward to write down the boundary data $\xi^{ \pm}$using the definition given in (22):

$$
\xi^{ \pm}=M^{ \pm}\left(\begin{array}{l}
V_{+} \\
V_{-}
\end{array}\right), \quad M^{ \pm} \equiv\left(\begin{array}{cc}
e^{ \pm i k L / 2} \sqrt{\omega-k} & -e^{\mp i k L / 2} \sqrt{\omega+k} \\
-e^{\mp i k L / 2} \sqrt{\omega+k} & e^{ \pm i k L / 2} \sqrt{\omega-k}
\end{array}\right)
$$

Hence the local boundary condition for the $1+1$ dimensional case with $\Omega=[-L / 2, L / 2]$ reads finally 


$$
\left(M^{-}-\mathbb{U} \gamma^{0} M^{+}\right)\left(\begin{array}{l}
V_{+} \\
V_{-}
\end{array}\right)=\left(\begin{array}{l}
0 \\
0
\end{array}\right),
$$

with $\mathbb{U} \gamma^{0}$ given by Equation (27). In order to have non-trivial solutions we must demand

$$
h(k) \equiv \operatorname{det}\left(M^{-}-\mathbb{U} \gamma^{0} M^{+}\right)=0 .
$$

After some algebraic manipulations on the last equation, we arrive at the secular equation for normal modes,

$$
h(k)=(\omega \cos \theta+m \cos \eta) \sin (k L)-k \sin \theta \cos (k L)=0,
$$

where $\theta \in[0,2 \pi]$, and $\eta \in[-\pi / 2, \pi / 2]$. Equivalently, Equation (35) can be written as

$$
\tan (k L)=\frac{k \sin \theta}{\omega \cos \theta+m \cos \eta} .
$$

The usual planar chiral bag boundary conditions for massive fermions correspond to $\theta=-\pi / 2$, $\eta=0$ [22]. Finally, let us mention that the bound state spectrum of the dual Casimir geometry is in all equivalent, but for the replacement of $L$ by $-L$ in the secular equation. Nonetheless, the spectrum of energies for $\omega>m$ contains in that case, in addition to discrete modes, a continuum of modes.

\subsection{Localised Edge States}

For massless fermions Equation (36) reads $\tan (k L)=\tan \theta$, and no localised states exist with $k$ purely imaginary. On the contrary, for $m>0$ a unique localised edge state may exist within the mass gap with $k= \pm i \kappa, 0<\kappa \leq m$. For this to be the case, it must hold that $[\omega \cos \theta+m \cos \eta] L / \sin \theta>1$. The equation for $q=\kappa / m$, with $0<q \leq 1$, is

$$
\tanh (q L m)=q \sin \theta /\left(\sqrt{1-q^{2}} \cos \theta+\cos \eta\right)
$$

From the last equation we can write

$$
\cos \eta=q \sin \theta \operatorname{coth}(L m q)-\sqrt{1-q^{2}} \cos \theta .
$$

The secular Equation (37) does not possess generally an analytical solution for $q$. However, if we take into account that $\eta \in[-\pi / 2, \pi / 2] \Rightarrow \cos \eta \in[0,1]$, we can obtain the regions of existence of edge states by representing the r.h.s. of (38), restricting ourselves to those areas in the domain $(q, \theta) \in[0,1] \times[0,2 \pi]$ such that $0<q \sin \theta \operatorname{coth}(L m q)-\sqrt{1-q^{2}} \cos \theta<1$. These regions are shown in Figure 1 . For the sake of illustration, we represent there the solutions of the above inequality for $m L=1$.

In conclusion, we have shown that the spectrum of vacuum fluctuations of a one-dimensional fermion confined in a filament of length $L$ under the boundary conditions of Equation (28) contains both propagating modes and localised edge states. Whereas the former obey Equation (36), the latter are given by Equation (37). The modes of the well-known planar chiral bag correspond to the boundary parameters $\theta=-\pi / 2, \eta=0$. 


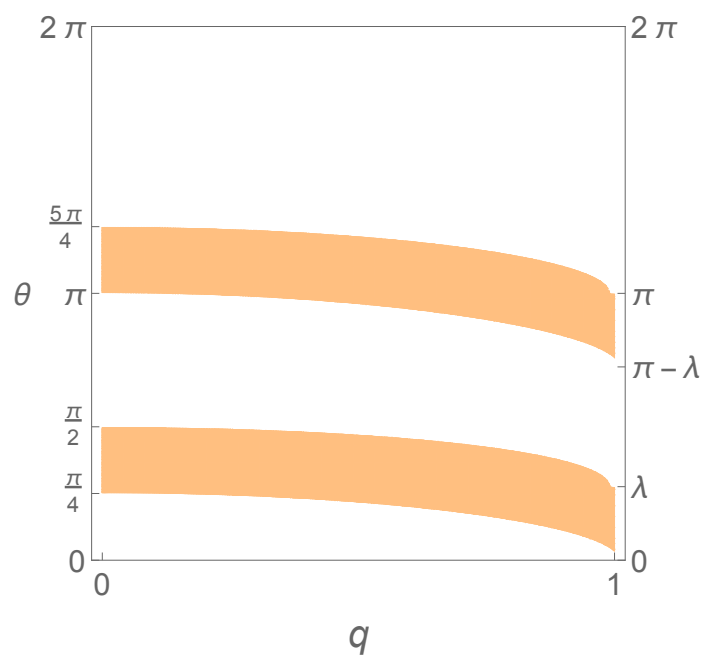

Figure 1. In light green, it is shown the region of boundary condition parameters for which edge states exist. Here $\lambda=\arcsin (\tanh 1)$, and $m L=1$.

\section{Casimir Energy of the Dirac Field in 1D}

The sum over the energies of the normal modes of the Dirac field in the Casimir geometry reads

$$
\mathcal{E}=2 \sum_{k \in \mathbb{R}^{+}}-\left|E_{k}\right|_{h(k)=0}=-\left.\sum_{k \in \mathbb{R}^{+}} 2 \sqrt{m^{2}+k^{2}}\right|_{h(k)=0}
$$

where the minus sign can be intended as the result of the negative energy of the Dirac sea.

The modes in Equation (39) are the real zeros of the secular Equation (36) that hereafter we refer to as $h(k)$. They depend implicitly on the parameters $\theta$ and $\eta$ of the corresponding unitary transformation. We apply Cauchy's argument principle to transform the infinite series of Equation (39) into a closed integral in the complex plane, with contour of integration the semiring of inner radius $m$ and outer infinite radius, with $\operatorname{Re}(k)>m$. In addition, we implement the regularisation scheme of Ref. [6] for odd spatial dimensions to arrive at—see also Refs. [23-25],

$$
\begin{aligned}
\mathcal{E} & =\frac{1}{2 \pi} \int_{m}^{\infty} d k k\left(1-m^{2} / k^{2}\right)^{\frac{1}{2}} \partial_{k} \log [h(i k, \omega(i k)) h(-i k,-\omega(-i k))]-\text { divergent terms } \\
& =\frac{1}{\pi} \int_{m}^{\infty} d k k\left(1-m^{2} / k^{2}\right)^{\frac{1}{2}} \operatorname{Re}\left(\partial_{k} \log [h(i k, \omega(i k))]\right)-\text { divergent terms, }
\end{aligned}
$$

where the lower limit of integration takes care of the restriction to propagating real modes out of the mass gap, and the subtraction of the divergent terms takes care of the ultraviolet (UV) regularisation of the integral. That regularisation consists of the discard of the divergent energies associated to the continuum of field fluctuations in free space ( $L$-independent terms) as well as of the infinite self-energy of the boundaries (terms proportional to $L$ ). Hereafter, for brevity we drop the dependence of $h$ with respect to $\omega(i k)$ in its argument. The factor $\partial_{k} \log [h(i k)]$ reads,

$$
\begin{aligned}
\partial_{k} \log [h(i k)]= & \frac{\cosh k L\left(-\sin \theta+m L \cos \eta+i L \cos \theta \sqrt{k^{2}-m^{2}}\right)}{-k \cosh k L \sin \theta+\sinh k L\left(m \cos \eta+i \cos \theta \sqrt{k^{2}-m^{2}}\right)} \\
& +\frac{\sinh k L\left(-k L \sin \theta+i k \cos \theta / \sqrt{k^{2}-m^{2}}\right)}{-k \cosh k L \sin \theta+\sinh k L\left(m \cos \eta+i \cos \theta \sqrt{k^{2}-m^{2}}\right)} .
\end{aligned}
$$


On the other hand, the UV divergent self-energy of the boundaries as $k \rightarrow \infty$ can be removed in Equation (40) by the subtraction of a term $L k\left(1-m^{2} / k^{2}\right)^{\frac{1}{2}}$ in the integrand, such that we end up with

$$
\mathcal{E}=\frac{1}{\pi} \int_{m}^{\infty} d k k\left(1-m^{2} / k^{2}\right)^{\frac{1}{2}} \operatorname{Re}\left(\partial_{k} \log [h(i k)]-L\right)-L \text {-independent divergent terms. }
$$

Formulas for the Casimir energy of massless and massive fermions in arbitrary dimensions were obtained in Refs. $[22,26,27]$ under planar chiral bag boundary conditions. In the following we compute the Casimir energy for general boundary conditions, restricting ourselves to the limit cases of light fields, $m L \ll 1$, and very massive fields, $m L \gg 1$.

\subsection{Casimir Energy of Light Fermions}

We consider first the light field limit, $m L \ll 1$. We will be interested here in the leading order mass corrections. From Equation (42) we observe that $\mathcal{E}$ contains three different terms, namely $\mathcal{E}=$ $\mathcal{E}_{0}+\mathcal{E}_{1}+\mathcal{E}_{2}$. In order to clarify the origin of each term, we first expand the integral around its lower limit of integration for $m L \ll 1$, up to $\mathcal{O}\left(m^{2}\right)$,

$$
\begin{aligned}
\mathcal{E} & =\frac{1}{\pi} \int_{m}^{\infty} d k k\left(1-m^{2} / k^{2}\right)^{\frac{1}{2}} \operatorname{Re}\left[\partial_{k} \log [h(i k)]-L\right] \\
& =\frac{1}{\pi} \operatorname{Re} \int_{0}^{\infty} d k k\left(1-m^{2} / k^{2}\right)^{\frac{1}{2}}\left[\partial_{k} \log [h(i k)]-L\right]-\frac{1}{\pi} \operatorname{Re} \int_{0}^{m} d k k\left(1-m^{2} / k^{2}\right)^{\frac{1}{2}}\left[\partial_{k} \log [h(i k)]-L\right] \\
& \left.\simeq \frac{1}{\pi} \operatorname{Re} \int_{0}^{\infty} d k k\left(1-m^{2} / k^{2}\right)^{\frac{1}{2}}\left[\partial_{k} \log [h(i k)]-L\right]\right|_{\mathcal{O}\left(m^{2}\right)^{\prime}}
\end{aligned}
$$

where the integral over the mass gap interval is purely imaginary and thus negligible, and we keep energy terms up to order $m^{2} L$ by expanding the integrand up to terms of order $m^{2}$. In addition, the extension of the lower limit of integration to zero compels us to discard the zero mode in $h(i k)$. Next, the integrand of the last equation must be expanded up to $\mathcal{O}\left(\mathrm{m}^{2}\right)$. Since the leading order expansion of the two factors, $k\left(1-m^{2} / k^{2}\right)^{\frac{1}{2}}$ and $\left[\partial_{k} \log [h(i k)]-L\right]$, is of order $m^{2}$ around $m=0$, we can distinguish three additive terms in $\mathcal{E}$, namely,

$$
\begin{aligned}
& \mathcal{E}_{0}=\frac{1}{\pi} \operatorname{Re} \int_{0}^{\infty} d k k\left[\partial_{k} \log [h(i k)]-L\right]_{m=0}, \\
& \mathcal{E}_{1}=\frac{1}{\pi} \operatorname{Re} \int_{0}^{\infty} d k k\left(1-m^{2} / k^{2}\right)_{\mathcal{O}\left(m^{2}\right)}^{\frac{1}{2}}\left[\partial_{k} \log [h(i k)]\right]_{m=0}, \\
& \mathcal{E}_{2}=\frac{1}{\pi} \operatorname{Re} \int_{0}^{\infty} d k k\left[\partial_{k} \log [h(i k)]\right]_{\mathcal{O}\left(m^{2}\right)} .
\end{aligned}
$$

The $m$-independent term is just the Casimir energy of a massless field, which depends on the parameter $\theta$,

$$
\mathcal{E}_{0}(\theta)=\frac{1}{2 \pi L} C_{2}(2 \theta) \text {. }
$$

Here, $C_{2}(2 \theta)$ is the generalised even Clausen function of order $2, C_{2}(2 \theta)=\sum_{n=1}^{\infty} \cos 2 n \theta / n^{2}$. In particular, for the usual planar chiral bag boundary conditions, $\theta=-\pi / 2$, we have $\mathcal{E}_{0}(-\pi / 2)=$ $-\pi / 24 L$, which coincides with the results of Ref. [26].

As for $\mathcal{E}_{1}$, once the first factor of the integrand of $\mathcal{E}_{1}$ is expanded at $\mathcal{O}\left(m^{2}\right)$, we get

$$
\mathcal{E}_{1}=\frac{-1}{2 \pi} \operatorname{Re} \int_{0}^{\infty} d k \frac{m^{2}}{k}\left[\partial_{k} \log [h(i k)]\right]_{m=0^{\prime}}
$$

which presents an infrared (IR) divergence for $k \rightarrow 0$. We can go around this problem by evaluating the associated discrete sum instead. That is, getting back to the expression of Equation (39) in one spatial dimension and taking $m=0$ in the secular equation, we have that the normal modes satisfy 
$\tan k_{n} L=\tan \theta$, from which $k_{n}=(n \pi+\theta) / L, n \in \mathbb{Z}-\{0\}$. Introducing these values into Equation (39) we obtain, up to $\mathcal{O}\left(m^{2}\right)$,

$$
\begin{aligned}
\mathcal{E} & =-2 \sum_{n=1} \sqrt{m^{2}+k_{n}^{2}}=-2 \sum_{n=1}^{\infty} \sqrt{m^{2}+\frac{(n \pi+\theta)^{2}}{L^{2}}} \simeq \frac{-2}{L} \sum_{n=1}^{\infty}(n \pi+\theta)\left[1+\frac{m^{2} L^{2}}{(n \pi+\theta)^{2}}\right] \\
& =\frac{-2}{L} \sum_{n=1}^{\infty}(n \pi+\theta)-\frac{m^{2} L}{\pi} \sum_{n=1}^{\infty} \frac{1}{n+\theta / \pi}=\frac{-2}{L} \sum_{n=1}^{\infty}(n \pi+\theta)-\frac{m^{2} L}{\pi} \sum_{n=0} \frac{1}{n+(1+\theta / \pi)} .
\end{aligned}
$$

The first term of the r.h.s. of the last equality is nothing but $\mathcal{E}_{0}(\theta)$, whereas we identify the second term with $\mathcal{E}_{1}(\theta)$. The infinite series of the latter term is the Hurwitz-zeta function $\zeta_{H}(s=1$, $q=1+\theta / \pi)$, whose analytical continuation contains a finite digamma function $\psi$ in addition to a simple pole of the form $1 /(s-1)$. The subtraction of the latter is meant to be our regularization prescription for $\mathcal{E}_{1}(\theta)$. With this proviso, we end up with the following finite expression (In the above equations we have assumed implicitly that $|\pi-\theta| \gg 0$. For $\theta \approx \pi$, a similar expansion is possible extracting the term $n=1$ out of the infinite series. By doing so one obtains the leading order $m, L$-dependent term $\mathcal{E}_{1}(\theta)=\frac{2 m^{2} L}{\pi} \psi(2+\theta / \pi)$ instead $)$

$$
\mathcal{E}_{1}(\theta)=\frac{m^{2} L}{\pi} \psi(1+\theta / \pi)
$$

As for $\mathcal{E}_{2}$, the expansion of $k \partial_{k} \log [h(i k)]$ contains a term of order $m L$ which, once integrated, yields a logarithmically divergent $L$-independent term which we discard in application of the regularisation prescription. In addition, the term of order $m^{2} L^{2}$ is

$$
\begin{aligned}
k \partial_{k} \log [h(i k)]= & \frac{m^{2} L^{2}}{4 y^{2} \sinh ^{3}(y+i \theta)}\left[2 y \cos \theta \sin ^{2} \theta \cosh y-2 \sin ^{2} \eta \cos \theta \sinh y\right. \\
& -\cos 2 \eta \cosh (2 y+i \theta) \sinh y+\cosh (2 y+3 i \theta) \sinh y-2 i y \sin \theta \sinh y],
\end{aligned}
$$

where $y=k L$. The above expression is a complicated function of $\theta$ whose integration needs of numerical methods. Nonetheless, in order to provide some insight into the contribution of this term to the Casimir energy, we consider in the following boundary conditions closed to those of the chiral bag. That is, we will expand the above expression up to leading order in $\delta$ with $\theta=-\pi / 2+\delta, \delta \ll 1$. The real part of the resultant expansion contains terms of order zero and two in $\delta$, which do not present any divergence neither in the UV nor in the IR. Its integration back in Equation (46) yields,

$$
\mathcal{E}_{2}(\delta, \eta) \simeq m^{2} L\left[0.28 \cos ^{2} \eta+0.18 \delta^{2}(1-2 \cos 2 \eta)\right], \quad \theta=-\pi / 2+\delta, \delta \ll 1 .
$$

The dominant contribution to the quantum vacuum energy for light fermions is given by (47), where $\mathcal{E}_{0}$ depends only on $\theta$. In Figure 2 we represent $\mathcal{E}_{0} L$ according to that equation.

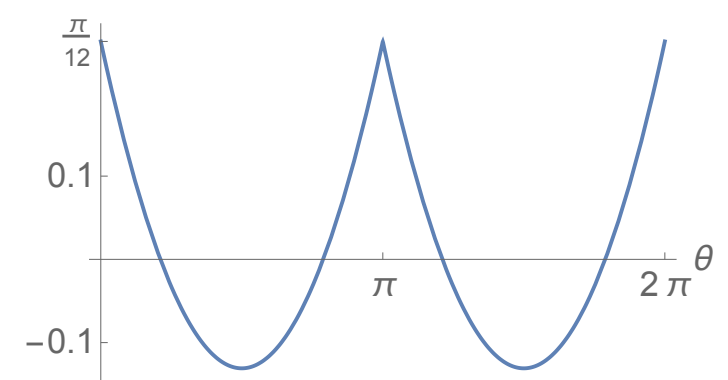

Figure 2. Plot of $\mathcal{E}_{0} L$ for massless fermions as a function of the self-adjoint extension parameter $\theta$, according to Equation (47). 


\subsection{Casimir Energy of Heavy Fermions}

As for the heavy fermion limit, $m L \gg 1$, we start with the regularised expression of Equation (42). For $m L \gg 1$ and $k \geq m, k L \gg 1$ holds in the whole range of integration. In this limit, the factor $\partial_{k} \log [h(i k)]-L$ within the integrand approaches

$$
\begin{aligned}
\operatorname{Re} \partial_{k} \log [h(i k)]-L \simeq & \frac{1}{2 m(y+\sin (\eta-\theta))}+\frac{1}{2 m(y-\sin (\eta+\theta))} \\
& +e^{-2 m L y} \frac{m L(\cos 2 \eta-\cos 2 \theta)+2 y^{2} m L \cos 2 \theta-\sin \theta \cos \eta}{2 m\left[y^{2}-2 y \sin \theta \cos \eta+\left(\cos ^{2} \eta-\cos ^{2} \theta\right)\right]}
\end{aligned}
$$

where $y=k / m \geq 1$. The first two terms on the r.h.s. of the last equation provide $L$-independent divergent terms when integrated in Equation (42), which we discard in accordance to our regularization prescription. The third term, together with the factor $m \sqrt{y^{2}-1}$, when integrated according to Equation (42) yields

$$
\mathcal{E} \simeq \frac{m}{2 \pi} e^{-2 m L} \int_{0}^{\infty} \mathrm{d} v e^{-2 m L v} \sqrt{v^{2}+2 v} \frac{-\sin \theta \cos \eta+m L\left[2(v+1)^{2} \cos 2 \theta+\cos 2 \eta-\cos 2 \theta\right]}{(v+1)^{2}-2(v+1) \sin \theta \cos \eta+\cos ^{2} \eta-\cos ^{2} \theta},
$$

where we have performed the change of variables $y=v+1$. In the limit $m L \gg 1$, the exponential factor suppresses the integrand for $v \leq 1 / m L \ll 1$. Therefore, we can expand the remainder of the integrand of the above equation in powers of $v$ which, in turn, implies an expansion of $\mathcal{E}$ in powers of $1 / \sqrt{m L}$. In particular, up to order $1 /(m L)^{3 / 2}$, we obtain

$$
\begin{aligned}
\mathcal{E} \simeq & \frac{m e^{-2 m L}}{8 \sqrt{\pi}\left(1-2 \sin \theta \cos \eta+\cos ^{2} \eta-\cos ^{2} \theta\right)}\left\{\frac{\cos 2 \theta+\cos 2 \eta}{\sqrt{m L}}\right. \\
& \left.+\frac{1}{(m L)^{3 / 2}}\left[-\sin \theta \cos \eta+\frac{3}{8} \cos 2 \eta+\frac{51}{8} \cos 2 \theta-\frac{3(1-\sin \theta \cos \eta)}{1-2 \sin \theta \cos \eta+\cos ^{2} \eta-\cos ^{2} \theta}\right]\right\} .
\end{aligned}
$$

Note that the parameters of the boundary conditions $\theta$ and $\eta$ can be chosen such that the leading order term vanishes. In particular, that is the case for $\theta= \pm \pi / 4, \eta= \pm \pi / 4$, and $\theta=\eta \pm \pi / 2$. Hence, the planar chiral bag boundary conditions fit into the latter case. In Figure 3 we show the behaviour of the the quantum vacuum energy (55) as a function of the parameters of the boundary condition. As can be seen, this energy can be positive, negative or zero. In addition, Figure 4 shows the behaviour of (55) as a function of $\theta$ for different values of the parameter $\eta$.

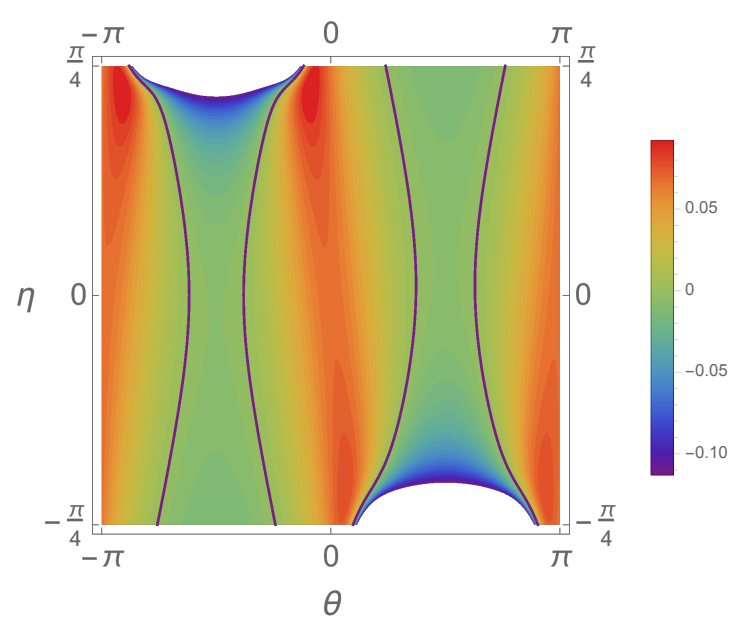

Figure 3. Plot of $2 \mathcal{E} /\left(m e^{-2 m L}\right)$ according to Equation (55) for $m L=20$, as a function of the self-adjoint parameters $\theta$ and $\eta$. The purple line represents the curve $\mathcal{E}=0$. Regions where $1-2 \sin \theta \cos \eta+$ $\cos ^{2} \eta-\cos ^{2} \theta$ is identically zero are excluded. 


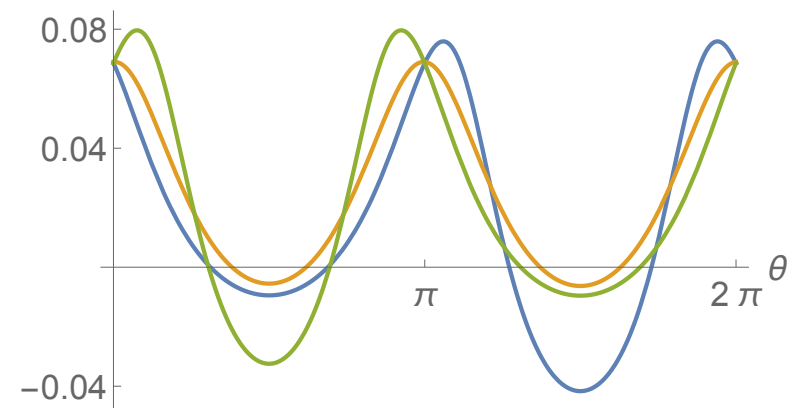

Figure 4. Plot of $2 \mathcal{E} /\left(m e^{-2 m L}\right)$ according to Equation (55) for $m L=20$, as a function of the self-adjoint extension parameter $\theta$, for different values of the parameter $\eta$. In blue, $\eta=-0.5$; in yellow, $\eta=0$; in green, $\eta=0.5$.

A Remark on the Sign of the Energy for Heavy Fermions

It is of special interest to consider the fermionic Casimir energy for identical "plates" and study its sign, in a similar way as it was done in [28]. Before doing so, we need to determine the configurations that can be understood as identical plates. The main result in reference [28] states that the sign on the Casimir energy is well defined when there is symmetry exchange between plates. Taking into account that in our field theory the plates are placed at $x= \pm L / 2$, the conditions of the central theorem in [28] will be satisfied by those boundary conditions that give rise to a parity-invariant quantum field theory. Since the free Dirac Hamiltonian is parity-invariant, all we need to know is which of the boundary conditions defined by Equation (25) are the ones that define parity-invariant field theories.

From [29] we know that the parity-transformed Dirac field mode $\psi$ in $1+1$ dimensional spacetime is given by

$$
\psi^{P}(x)=\gamma^{0} \psi(-x) .
$$

From this transformation and under the representation we have taken for the gamma matrices, it is straightforward to write the boundary spinors for the parity-transformed field,

$$
\xi_{P}^{ \pm}=\gamma^{0} \xi^{\mp} .
$$

Using now Equation (23), we can write the boundary condition for the spinor $\psi^{P}(x)$ in terms of its corresponding boundary spinors $\xi_{P}^{ \pm}$,

$$
\xi_{P}^{-}=\mathbb{U}^{-1} \gamma^{0} \xi_{P}^{+}=\mathbb{U}^{\dagger} \gamma^{0} \xi_{P}^{+} .
$$

Parity-invariant boundary conditions will be given by those that remain invariant under the parity transformation, i.e., that give rise to the same boundary condition for the spinor $\psi^{P}(x)$. Hence, in our case parity-invariant boundary conditions are given by those unitary matrices in (25) that satisfy $\mathbb{U}^{+}=\mathbb{U}$. This restriction imposes $\theta \pm \eta=\pi \mathbb{Z}$, and taking into account that $(\theta, \eta) \in[0,2 \pi] \times[-\pi / 2, \pi / 2]$ the unitary matrices that give parity-invariant field theories are finally given by

$$
\theta= \pm \eta ; \quad \theta=\pi \pm \eta ; \quad \theta=2 \pi \pm \eta .
$$

As can be seen in Figure 5, the sign of the quantum vacuum energy is not conserved for parity-invariant boundary conditions. It is of note that the same change of sign is observed for the energy of massless fermions for parity-invariant boundary conditions. 


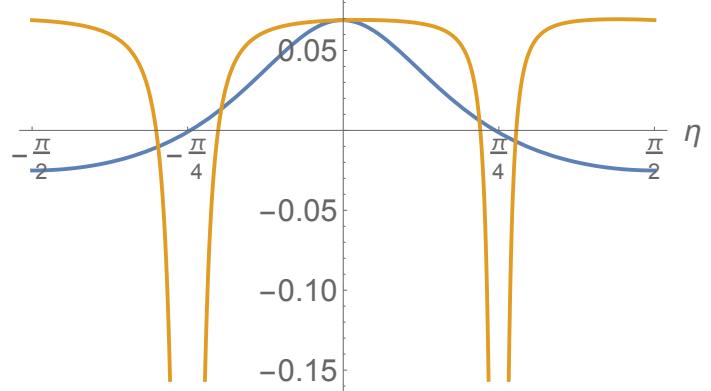

Figure 5. Plot of $2 \mathcal{E} /\left(m e^{-2 m L}\right)$ according to Equation (55) for $m L=20$. The blue curve corresponds to $\theta=\eta+\pi$ with $\eta \in[-\pi / 2, \pi / 2]$, to $\theta=-\eta+2 \pi$ with $\eta \in[0, \pi / 2]$, and to $\theta=-\eta$ with $\eta \in[-\pi / 2,0]$. The orange curve corresponds to $\theta=-\eta+\pi$ with $\eta \in[-\pi / 2, \pi / 2]$, for $\theta=\eta$ with $\eta \in[0, \pi / 2]$, and for $\theta=\eta+2 \pi$ with $\eta \in[-\pi / 2,0]$.

In addition, taking into account Figure 2 and the conditions (59), it is easy to see that since $\mathcal{E}_{0}$ in (47) does not depend on $\eta$, the sign of the quantum vacuum energy does not remain fixed for parity-invariant self-adjoint extensions in the light fermions case. Therefore, we can conclude with the following theorem that summarizes one of the main results of this paper:

Theorem 1. Parity-invariant self-adjoint extensions of the $1 D$ Dirac operator give rise to $1+1$ dimensional quantum field theories where the sign of the quantum vacuum energy can be positive or negative.

\section{Conclusions and Outlook}

We have studied the self-adjoint extensions of the one-dimensional Dirac operator for massive fermions confined in a filament of length $L$, as well as in its dual geometry. The construction of the space of boundary spinors has been formulated in detail for the case of global and stationary boundary conditions. The spectrum of vacuum field fluctuations has been analysed as a function of the boundary condition parameters. It is found that, for a massive field, it is possible to find an edge state within the mass gap. However, its region of existence in the space of boundary parameters is bounded by Equation (37). As for the spectrum of discrete normal modes, we have computed its Casimir energy. Our regularisation scheme allows us to obtain finite results for the limit regimes of light fermions, $m L \ll 1$, and heavy fermions, $m L \gg 1$, for general boundary conditions. In the former case, we obtain analytical results up to terms of order $m^{2} L$ around the planar chiral bag conditions-Equations (47), (50) and (52). In the latter case, we compute terms up to order $m e^{-2 m L} /(m L)^{3 / 2}$-Equation (55). In either regime, the graphical representations in Figures $2-4$ show that both positive and negative Casimir energies can be obtained with different boundary parameters. For a fixed mass, the absolute value of the energy decreases monotonically with the distance. Therefore, attractive forces are obtained for negative energies whereas repulsive forces hold for positive energies in both regimes. Interestingly, it is found that the sign of the energy is not conserved for parity-invariant boundary conditions (see Figure 5). This result is in contrast to the one obtained in Ref. [28] for scalar and electromagnetic fields, where it was found that parity-invariant extensions of the Laplace operator ensure a fixed sign of the vacuum energy. Hence, it follows that in fermionic Casimir effect we cannot ensure that "opposites repeal" or "opposites attract", as it happens for electromagnetic and scalar Casimir effects, where "opposites always attract".

The self-adjoint extensions analyzed in this paper correspond to those arising from local boundary conditions that have a clear classical analogue, and thus can be implemented in a path integral approach (see Ref. [30]). It is worth mentioning that the case of global boundary conditions for the dual Casimir geometry includes as a limit case the Dirac- $\delta$ for Dirac fields as $L \rightarrow 0[29,31]$. The case of global boundary conditions involving changes in the topology of the boundary, in the sense of Ref. [32], is left for future work. 
Author Contributions: All the authors have contributed equally to this work.

Funding: Financial support from grants MTM2014-57129-C2-1-P (MINECO), VA057U16 and BU229P18 (Junta de Castilla y León and FEDER), and VA137G18 (Junta de Castilla y León), is gratefully acknowledged. M.T.-F. acknowledges the financial support of the European Social Fund, the Operational Programe of Junta de Castilla y León and the regional Ministry of Education.

Conflicts of Interest: The authors declare no conflict of interest.

\section{References}

1. Asorey, M.; Ibort, A.; Marmo, G. Global theory of quantum boundary conditions and topology change. Int. J. Mod. Phys. A 2005, 20, 1001. [CrossRef]

2. Grubb, G. A characterization of the non-local boundary value problems associated with an elliptic operator. Ann. Sc. Norm. Super. Pisa. 1968, 22, 425.

3. Asorey, M.; Garcia-Alvarez, D.; Munoz-Castaneda, J.M. Casimir Effect and Global Theory of Boundary Conditions. J. Phys. A Math. Gen. 2006, 39, 6127. [CrossRef]

4. Asorey, M.; Garcia-Alvarez, D.; Munoz-Castaneda, J.M. Vacuum Energy and Renormalization on the Edge. J. Phys. A Math. Theor. 2007, 40, 6767. [CrossRef]

5. Asorey, M.; Munoz-Castaneda, J.M. Vacuum Boundary Effects. J. Phys. A Math. Theor. 2008, 41, 304004. [CrossRef]

6. Asorey, M.; Munoz-Castaneda, J.M. Attractive and Repulsive Casimir Vacuum Energy with General Boundary Conditions. Nucl. Phys. B 2013, 874, 852. [CrossRef]

7. Silva, H.O.; Farina, C. A Simple model for the dynamical Casimir effect for a static mirror with time-dependent properties. Phys. Rev. D 2011, 84, 045003. [CrossRef]

8. Milton, K.A. The Casimir Effect: Physical Manifestations of Zero-Point Energy; World Sci.: Singapore, 2001.

9. Milonni, P.W. The Quantum Vacuum; Academic Press: San Diego, CA, USA, 1994.

10. Ibort, A.; Lledó, F.; Pérez-Pardo, J.M. On Self-Adjoint Extensions and Symmetries in Quantum Mechanics. Ann. Henri Poincaré 2015, 1460, 15-54. [CrossRef]

11. Ibort, A. Three lectures on global boundary conditions and the theory of self-adjoint extensions of the covariant Laplace-Beltrami and Dirac operators on Riemannian manifolds with boundary AIP Conf. Proc. 2012, 16, 2367-2397.

12. Pérez-Pardo, J.M.; Barbero-Linán, M.; Ibort, A. Boundary dynamics and topology change in quantum mechanics. Int. J. Geom. Methods Mod. Phys. 2015, 12, 1560011. [CrossRef]

13. Facchi, P.; Garnero, G.; Ligabo, L. Quantum cavities with alternating boundary conditions. J. Phys. A Math. Theor. 2018, 51, 105301. [CrossRef]

14. Facchi, P.; Garnero, G.; Marmo, G.; Samuel, J.; Sinha, S. Boundaries without boundaries. Ann. Phys. 2018, 394, 139. [CrossRef]

15. Asorey, M.; Balachandran, A.P.; Pérez-Pardo, J.M. Edge states at phase boundaries and their stability. Rev. Math. Phys. 2016, 28, 1650020. [CrossRef]

16. Chodos, A.; Jaffe, R.L.; Johnson, K.; Thorn, C.B.; Weisskopf, V.F. New Extended Model of Hadrons. Phys. Rev. D 1974, 9, 3471. [CrossRef]

17. Johnson, K. The M.I.T. Bag Model. Acta Phys. Polon. B 1975, 6, 865.

18. Milton, K.A. Fermionic Casimir Stress on A Spherical Bag. Ann. Phys. 1983, 150, 432. [CrossRef]

19. Elizalde, E.; Bordag, M.; Kirsten, K. Casimir energy for a massive fermionic quantum field with a spherical boundary. J. Phys. A 1998, 31, 1743. [CrossRef]

20. Asorey, M.; Ibort, A.; Marmo, G. The topology and geometry of self-adjoint and elliptic boundary conditions for Dirac and Laplace operators. Int. J. Geom. Methods Mod. Phys. 2015, 12, 1561007. [CrossRef]

21. Balachandran, A.P.; Bimonte, G.; Marmo, G.; Simoni, A. Topology change and quantum physics. Nucl. Phys. B 1995, 446, 299. [CrossRef]

22. Mamaev, S.G.; Trunov, N.N. Vacuum expectation values of the energy-momentum tensor of quantized fields on manifolds with different topologies and geometries. III. Sov. Phys. 1980, 23, 551. [CrossRef]

23. Vassilevich, D.V. Heat kernel expansion: User's manual. Phys. Rep. 2003, 388, 279-360. [CrossRef]

24. Blau, S.K.; Visser, M.; Wipf, A. Zeta functions and the Casimir energy. Nucl. Phys. B 1988, 310, $163-180$. [CrossRef] 
25. Kirsten, K. Spectral Functions in Mathematics and Physics; Chapman and Hall/CRC: Boca Raton, FL, USA, 2001.

26. De Paola, R.D.M.; Rodrigues, R.B.; Svaiter, N.F. Casimir Energy of Massless Fermions in the Slab-bag. Mod. Phys. Lett. A 1999, 14, 2353. [CrossRef]

27. Elizalde, E.; Santos, F.C.; Tort, A.C. The Casimir Energy of a Massive Fermionic Field Confined in a $(\mathrm{d}+1)$-dimensional Slab-Bag. Int. J. Mod. Phys. A 2003, 18, 1761. [CrossRef]

28. Kenneth, O.; Klich, I. Opposites attract: A Theorem about the Casimir force. Phys. Rev. Lett. 2006, 97, 160401. [CrossRef]

29. Mateos-Guilarte, J.; Munoz-Castaneda, J.M.; Pirozhenko, I.; Santamaria-Sanz, L. One-dimensional scattering of fermions on $\delta$-impurities. arXiv 2019, arXiv:1903.05568v2.

30. Asorey, M.; Clemente-Gallardo, J.; Munoz-Castaneda, J.M. Path integrals and boundary conditions. J. Phys. Conf. Ser. 2007, 87, 012004. [CrossRef]

31. Sundberg, P.; Jaffe, R.L. The Casimir effect for fermions in one-dimension. Ann. Phys. (N. Y.) 2004, $309,442$. [CrossRef]

32. Asorey, M.; Ibort, A.; Marmo, G. Path integrals and boundary conditions. In Proceedings of the Meeting on Fundamental Physics 'A. Galindo', Madrid, Spain, 26 November 2004.

(C) 2019 by the authors. Licensee MDPI, Basel, Switzerland. This article is an open access article distributed under the terms and conditions of the Creative Commons Attribution (CC BY) license (http:/ / creativecommons.org/licenses/by/4.0/). 